\title{
The value of screening for cognition, depression, and frailty in patients referred for TAVI
}

This article was published in the following Dove Press journal:

Clinical Interventions in Aging

\author{
Maisha M Khan ${ }^{1,2}$ \\ Krista L Lanctôt ${ }^{1-3}$ \\ Stephen E Fremes ${ }^{4}$ \\ Harindra C Wijeysundera ${ }^{4}$ \\ Sam Radhakrishnan ${ }^{4}$ \\ Damien Gallagher ${ }^{3}$ \\ Dov Gandell ${ }^{5}$ \\ Megan C Brenkel ${ }^{1}$ \\ Elias L Hazan' \\ Natalia G Docteur' \\ Nathan Herrmann ${ }^{1,3}$ \\ 'Neuropsychopharmacology Research \\ Group, Hurvitz Brain Sciences Program, \\ Sunnybrook Research Institute, Toronto, \\ Ontario, Canada; ${ }^{2}$ Department of \\ Pharmacology and Toxicology, University \\ of Toronto, Toronto, Ontario, Canada; \\ ${ }^{3}$ Department of Psychiatry, Sunnybrook \\ Health Sciences Centre and University of \\ Toronto, Toronto, Ontario, Canada; \\ ${ }^{4}$ Schulich Heart Centre, Sunnybrook \\ Health Sciences Centre, Toronto, \\ Ontario, Canada; ${ }^{5}$ Department of \\ Geriatric Medicine, Sunnybrook Health \\ Sciences Centre, Toronto, Ontario, \\ Canada
}

Correspondence: Nathan Herrmann Lewar Chair in Geriatric Psychiatry Head, Division of Geriatric Psychiatry Sunnybrook Health Sciences Centre, 2075 Bayview Ave, Room FG 19, Toronto M4N 3M5 Ontario, Canada

Tel + I 4164806133

$\mathrm{Fax}+$ I 4164806022

Email Nathan.Herrmann@sunnybrook.ca
Background: Current surgical risk assessment tools fall short of appreciating geriatric risk factors including cognitive deficits, depressive, and frailty symptoms that may worsen outcomes post-transcatheter aortic valve implantation (TAVI). This study hypothesized that a screening tool, SMARTIE, would improve detection of these risks pre-TAVI, and thus be predictive of postoperative delirium (POD) and 30-day mortality post-TAVI.

Design: Prospective observational cohort study, using a historical cohort for comparison. Participants: A total of 234 patients (age: $82.2 \pm 6.7$ years, $59.4 \%$ male) were included. Half were screened using SMARTIE.

Methods: The SMARTIE cohort was assessed for cognitive deficits and depressive symptoms using the Mini-Cog test and PHQ-2, respectively. Measures of frailty included activities of daily living inventory, the Timed Up and Go test and grip strength. For the pre-SMARTIE cohort, we extracted cognitive deficits, depression and frailty symptoms from clinic charts. The incidence of POD and 30-day mortality were recorded. Bivariate chi-square analysis or $t$-tests were used to report associations between SMARTIE and pre-SMARTIE groups. Multivariable logistic regression models were employed to identify independent predictors of POD and 30-day mortality.

Results: More patients were identified with cognitive deficits $\left(\chi^{2}=11.73, p=0.001\right)$, depressive symptoms $\left(\chi^{2}=8.15, p=0.004\right)$, and physical frailty $\left(\chi^{2}=5.73, p=0.017\right)$ using SMARTIE. Cognitive deficits were an independent predictor of POD (OR: 8.4, $p<0.01$ ) and 30-day mortality (OR: 4.04, $p=0.03$ ).

Conclusion: This study emphasized the value of screening for geriatric risk factors prior to TAVI by demonstrating that screening increased identification of at-risk patients. It also confirmed findings that cognitive deficits are predictive of POD and mortality following TAVI.

Keywords: TAVI, cognition, depression, frailty

\section{Introduction}

Transcatheter aortic valve implantation (TAVI) is increasingly preferred for treating severe aortic stenosis in patients at risk of surgical complications due to advanced age and associated comorbidities. ${ }^{1,2}$ It has been shown to reduce mortality and improve both quality of life and functional status in older patients with aortic stenosis. ${ }^{3}$ TAVI is superior to medical therapy and similar to the current standard of care, surgical valve replacement (SAVR), although it has also been associated with lower 12-month mortality rates compared to SAVR. ${ }^{1,2,4}$

TAVI was initially recommended primarily in patients at highest risk, but now treatment is shifting to intermediate and even low-risk patients. ${ }^{5,6}$ In the current clinical practice, surgical risk is evaluated using risk model algorithms developed 
by the European System for Cardiac Operative Risk Evaluation score (EuroScore) II and Society of Thoracic Surgeons score. ${ }^{7,8}$ While those traditional risk scores account for a number of risk factors including age, gender, symptomatic disease status, previous cardiac surgery, recent myocardial infarction, cardiovascular risk factors, renal dysfunction, mobility and procedure urgency, important risk factors that are prevalent in geriatric populations such as cognitive impairment, mood disorders, and physical frailty are not included. ${ }^{7,8}$ A prospective cohort study has recently highlighted the need for the development of improved risk prediction models using multidimensional geriatric assessments in TAVI. ${ }^{9}$

The majority of older patients hospitalized for acute cardiac conditions present with at least one major geriatric syndrome: frailty, cognitive deficits, severe dependence, and depression, that have been associated with poorer inhospital and post-discharge functional and clinical outcomes. ${ }^{10}$ Pre-existing cognitive deficits are an important consideration prior to cardiac intervention as they have been associated with poor outcomes in TAVI patients. ${ }^{11}$ Cognitive deficits and associated comorbidities have also been shown to have an additive effect on mortality, resulting in poor survival rates. ${ }^{12}$ Notably, a recent meta-analysis on cognitive outcomes following surgery for valvular heart disease found that patients were at risk for cognitive decline over the six months after surgery. ${ }^{13}$ Depression is also common in older adults and a major public health problem because of its related morbidity and excess mortality. ${ }^{14}$ Depression has been associated with cognitive deficits in geriatric out-patients as well as patients with heart disease. ${ }^{14-17} \mathrm{~A}$ recent paper examined the effect of depression on 30-day mortality in post-TAVI and SAVR patients and found that depression was significantly associated with mortality. Depressed patients were also more likely to have comorbid frailty and cognitive deficits than their non-depressed counterparts. ${ }^{17}$ Physical functional status is similarly an important determinant of independent living and long-term outcomes, and several studies have identified physical frailty as a predictor of poor outcomes after TAVI. ${ }^{18-20}$ A systematic review on the relationship between preoperative frailty status and TAVI outcomes also suggests that frailty is associated with increased mortality in this vulnerable population. ${ }^{21}$

Despite evidence suggesting the importance of geriatric risk factors in predicting outcomes following cardiac intervention, current surgical risk assessment falls short of appreciating true risks among TAVI patients. These factors may be particularly important when assessing a geriatric, multi-morbid population who may present with subtle cognitive deficits, depressive and frailty symptoms that may increase the risk of poor TAVI outcomes. This study aimed to use a brief screening tool to identify TAVI patients with the specific geriatric risk factors noted above and assess the impact of these factors on poor postTAVI outcomes. We proposed that 1) the screen would improve the detection of the geriatric-specific risk factors of cognitive deficits, depressive symptoms, and physical frailty among TAVI patients and 2) that these geriatric symptoms ie, cognitive deficits, depressive and frailty symptoms, would be predictive of postoperative delirium (POD) and 30-day mortality.

\section{Methods}

This was a prospective observational cohort study, using a historical cohort for comparison. A screening tool (SMARTIE: Screening for and MAnaging Risk factors in TAVI: an Interdisciplinary Endeavour) was developed to identify geriatric symptoms including cognitive deficits, depressive symptoms, and physical frailty. Englishspeaking patients referred for TAVI were screened using SMARTIE at Sunnybrook Health Sciences Centre (Sunnybrook) in Toronto, Ontario, Canada from September 2015 to August 2017. SMARTIE was administered by the attending physician or nurse during initial assessment for TAVI at Sunnybrook and took approximately 10 mins to administer. Cognitive deficits were assessed using the Mini-Cog test, a brief instrument consisting of an un-cued three-item recall test for memory and a simply scored clock drawing test that has been used to detect cognitive deficits in older adults. ${ }^{22}$ Patients who were able to recall less than three words and who had an abnormal clock were scored positive for cognitive deficits. ${ }^{23}$ Depressive symptoms were assessed using the Patient Health Questionnaire (PHQ-2), a valid screening tool for major depression in older people. ${ }^{24}$ A cut-off score of three was considered positive for depressive symptoms. ${ }^{25}$ Measures of frailty included the activities of daily living (ADL) inventory, the Timed Up and Go (TUG) test and measure of grip strength, where those with deficits in at least one test were scored positive for frailty. ${ }^{26,27}$ The ADL inventory measures the activities needed to live independently and the extent to which respondents are able to perform these activities on their own. The TUG test is a mobility assessment that evaluates patients' pace, balance, and timing in a walking exercise. 
Lastly, grip strength measures the average force $(\mathrm{kg})$ that a patient can use in both the dominant and non-dominant hand. ${ }^{27}$ However, not all screened patients were eligible for, or completed TAVI (eg, asymptomatic aortic stenosis patients, other medical conditions barring intervention) during the mentioned time period. Therefore, only patients who had been screened and completed a TAVI procedure were included in the SMARTIE cohort.

For the pre-SMARTIE historical cohort comparison group, a retrospective chart review was conducted for patients who completed TAVI between September 2014 and August 2015, which corresponded to an observation period immediately before the SMARTIE screen was implemented. The retrospective chart review was performed at Sunnybrook by three of the authors (MK, EH, $\mathrm{MB})$. Retrospective data were recorded systematically on a standardized template to capture all target data points and reduce the risk of unintended bias in data collection. Demographic and clinical characteristics including age, sex, clinical diagnoses, medication use and the number of referrals to Geriatric Psychiatry were extracted. Clinical charts were also reviewed for record of subjective complaints of cognitive deficits, depressive symptoms, and frailty as indicators of geriatric symptoms prior to TAVI. Additionally, data for post-procedural outcomes such as hospital length of stay, discharge from hospital, readmissions, POD, mortality, and vascular complications postTAVI were collected from chart review for all patients in both study cohorts.

\section{Study outcomes}

Our primary objective was to compare detection of geriatric symptoms (primary outcome) including cognitive deficits, depressive symptoms, and physical frailty in patients prior to receiving TAVI in the SMARTIE and preSMARTIE cohorts. Our secondary objective was to assess the relationship between these symptoms and occurrences of POD and 30-day mortality (secondary outcomes).

The proportion of patients with geriatric symptoms of cognitive deficits, depressive symptoms, and frailty prior to TAVI were recorded in both the SMARTIE and preSMARTIE groups for comparison. Data on post-TAVI POD and 30-day mortality were extracted from hospital charts by three authors (MK, EH, MB) for all patients. Delirium was assessed using the Intensive Care Delirium Screening checklist by an attending physician or nurse practitioner in the Intensive Care Unit. It is a validated tool to screen for delirium in cardiac surgery patients with high sensitivity and specificity consisting of an 8-item checklist. One point is allotted for an abnormality in each item on the list and a total score $\geq 4$ is classified as positive for delirium. ${ }^{28}$ POD was differentiated from other neurological events early after TAVI. In the case of concern of clinical stroke, a CT or MRI scan would follow as clinically relevant.

\section{Data analyses}

Data analyses were performed using IBM SPSS Statistics. ${ }^{24}$ Baseline demographics and clinical characteristics between the SMARTIE and pre-SMARTIE groups were compared using bivariate chi-square analysis for categorical data or $t$-tests for continuous data. Chi-square analyses were used to compare the proportion of patients with geriatric symptoms including cognitive deficits, depressive symptoms, and frailty between the SMARTIE and pre-SMARTIE groups. In addition, multivariable logistic regression models were employed to assess the relationship between geriatric symptoms and outcomes post-TAVI including POD and 30-day mortality. These models included both pre-SMARTIE and SMARTIE patients. Cognitive deficits, depressive, and frailty symptoms were added as independent predictors to multivariable models predicting the likelihood of POD and 30-day mortality. All models adjusted for age and general anesthesia as they have been previously associated with poor TAVI outcomes. ${ }^{29,30}$ Multicollinearity was assessed before inclusion of all predictors into the model and the variable that was most strongly related in a colinear set was added to the model. Risk of outcomes associated with geriatric symptoms was presented as an odds ratio and 95\% confidence interval. The fit of the prediction model was assessed by computing the Hosmer-Lemeshow goodnessof-fit test where $p>0.05$ refers to a good fit model. All analyses were two-tailed, and a $p$-value $<0.05$ was accepted as significant.

\section{Results}

\section{Patient characteristics}

A total of 234 older adults (age: $82.2 \pm 6.7$ years, 59.4\% male) were included in this study. Geriatric symptoms were identified prospectively using SMARTIE in 117 TAVI patients and retrospectively using chart review in 117 TAVI patients before SMARTIE was implemented (Table 1). The pre-SMARTIE cohort was slightly younger, more likely to undergo general anesthesia during the TAVI 
Table I Baseline characteristics and outcomes in patients before and after the SMARTIE screen was implemented

\begin{tabular}{|c|c|c|c|c|}
\hline & Pre-SMARTIE $(n=|| 7)$ & SMARTIE $(n=|| 7)$ & $\chi^{2}$ or $t$ & $p^{*}$ \\
\hline Age, mean $\pm S D$ & $81.2 \pm 7.4$ & $83.3 \pm 5.7$ & -2.45 & $0.015^{*}$ \\
\hline Gender (Male), n(\%) & $68(58.1)$ & $71(60.7)$ & 0.16 & 0.69 \\
\hline \multicolumn{5}{|l|}{ Screening outcomes } \\
\hline Cognitive deficits, n(\%) & $19(16.2)$ & $42(35.9)$ & 11.73 & $0.001 *$ \\
\hline Depressive symptoms, $n(\%)$ & $5(4.3)$ & $18(15.4)$ & 8.15 & $0.004^{*}$ \\
\hline Frailty symptoms, $n(\%)$ & $6(5.1)$ & $17(14.5)$ & 5.73 & $0.017^{*}$ \\
\hline \multicolumn{5}{|l|}{ TAVI characteristics } \\
\hline General anesthesia, $\mathrm{n}(\%)^{\ddagger}$ & $116(99.1)$ & $27(23.1)$ & 142.4 & $<0.00 I^{*}$ \\
\hline TAVI urgency, $n(\%){ }^{\#}$ & $21(17.9)$ & $12(10.3)$ & 2.86 & 0.09 \\
\hline \multicolumn{5}{|l|}{ Procedural outcomes } \\
\hline Postoperative delirium, n(\%) & $16(13.7)$ & $7(6.0)$ & 3.91 & $0.048^{*}$ \\
\hline Stroke/transient ischemic attack, $\mathrm{n}(\%)$ & $4(3.4)$ & $7(6.0)$ & 0.86 & 0.35 \\
\hline Procedural complications, n(\%) & $22(18.8)$ & $24(20.5)$ & 0.11 & 0.74 \\
\hline Hospital length of stay, mean $\pm S D$ & $11.5 \pm 23.7$ & $5.4 \pm 6.4$ & 2.67 & $0.008^{*}$ \\
\hline 30-day mortality, n(\%) & $9(7.7)$ & $4(3.4)$ & 2.04 & 0.15 \\
\hline
\end{tabular}

Notes: $\neq$ General anesthesia compared to the use of local anesthesia with conscious sedation. ${ }^{\#}$ Urgent TAVI (including emergency and salvage surgery) compared the preplanned procedure. * $p$ significance: $p<0.05$.

Abbreviation: TAVI, transcatheter aortic valve implantation.

procedure, and had a higher incidence of POD and longer length of stay in hospital post-TAVI compared to the SMARTIE cohort (Table 1).

\section{Detection of geriatric symptoms in SMARTIE vs pre-SMARTIE cohorts}

All 117 patients in the SMARTIE cohort were assessed for cognitive deficits and depressive symptoms, however, nine patients were not assessed for frailty symptoms. For the pre-SMARTIE cohort, all 117 patient charts were reviewed for recorded evidence of cognitive deficits, depressive symptoms, and frailty, although one patient chart could not be reviewed for frailty. Among those screened with SMARTIE, cognitive deficits were the most common geriatric symptom, followed by depressive symptoms and frailty symptoms. The proportions of patients with cognitive deficits $(35.9 \%$ vs $16.2 \%$; $\left.\chi^{2}=11.73, p=0.001\right)$, depressive symptoms $(15.4 \%$ vs $\left.4.3 \% ; \chi^{2}=8.15, p=0.004\right)$, and physical frailty $(14.5 \%$ vs $\left.5.1 \% ; \chi^{2}=5.73, p=0.017\right)$ prior to TAVI were significantly higher in the SMARTIE cohort compared to the preSMARTIE cohort (Table 1). Among the subgroup of SMARTIE patients with geriatric symptoms who were referred for a geriatric specialist consultation following the screen, $59.0 \%$ were diagnosed with mild cognitive impairment or dementia, while $91.3 \%$ and $100 \%$ were diagnosed with depression and frailty, respectively.

\section{Association between geriatric symptoms and POD in TAVI}

In total 23/234 patients (9.8\%) experienced delirium postTAVI. In a model adjusted for age and the use of anesthesia, cognitive deficits (OR: 8.4, $p<0.01$ ), but not depressive symptoms or frailty, were an independent predictor of POD (Table 2). This model showed a good fit $\chi^{2}=3.34$, $p=0.91)$.

\section{Association between geriatric symptoms and 30-day mortality in TAVI}

The total mortality rate 30 days post-TAVI was $5.6 \%$ in this study. In a model adjusting for age and the use of anesthesia, cognitive deficits (OR: 4.04, $p=0.03$ ) predicted 30-day mortality while depressive and frailty symptoms did not (Table 3). This model showed a good fit $\left(\chi^{2}=3.78, p=0.88\right)$.

\section{Discussion}

This study incorporated a novel screening tool as part of the TAVI clinical care pathway to improve peri-procedural risk management. The screening tool provided additional health status information of geriatric-specific risk factors in TAVI patients including cognitive deficits, depressive symptoms, and physical frailty. In the past few years, there has been increased research emphasizing the importance of multi geriatric assessment (MGA) of TAVI patients 
Table 2 Predictors of postoperative delirium (POD)

\begin{tabular}{|c|c|c|c|c|c|c|c|}
\hline & $\boldsymbol{\beta}$ & Standard error & Wald & $D f$ & $\mathbf{p}^{*}$ & $\begin{array}{l}\text { Odds } \\
\text { ratio }\end{array}$ & $95 \% \mathrm{Cl}$ for Odds ratio \\
\hline Cognitive deficits & 2.13 & 0.54 & $|5.5|$ & 1.00 & $<0.0 I^{*}$ & 8.44 & $2.92-24.38$ \\
\hline Depressive symptoms & -1.74 & 1.25 & 1.93 & 1.00 & 0.17 & 0.18 & $0.02-2.05$ \\
\hline Frailty symptoms & 1.47 & 0.76 & 3.73 & 1.00 & 0.05 & 4.35 & $0.98-19.31$ \\
\hline Age & 0.09 & 0.05 & 3.45 & 1.00 & 0.06 & 1.09 & $1.00-1.19$ \\
\hline General anesthesia $^{\#}$ & 2.91 & 0.83 & 12.39 & 1.00 & $<0.0 I^{*}$ & 18.37 & $3.63-92.89$ \\
\hline Constant & $-|2.6|$ & 4.09 & 9.50 & 1.00 & $<0.01$ & 0.00 & \\
\hline
\end{tabular}

Notes: \#Compared to local anesthesia under conscious sedation. * $p$ significance, $p<0.05$. Without SMARTIE: The model $\left(\chi^{2}(5)=39.2\right.$, $\left.p<0.00 \mathrm{I}\right)$ explained $33.3 \%($ Nagelkerke $R^{2}$ ) of the variance in predicting POD correctly classifying $91.1 \%$ of POD cases. The Hosmer \& Lemeshow test of the goodness of fit suggested the model was a good fit to the data $(p=0.91)$.

Table 3 Predictors of 30-day mortality rate

\begin{tabular}{|l|l|l|l|l|l|l|l|}
\hline & $\boldsymbol{\beta}$ & Standard error & Wald & Df & $\boldsymbol{P}^{*}$ & $\begin{array}{l}\text { Odds } \\
\text { ratio }\end{array}$ & 95\% Cl for Odds ratio \\
\hline Cognitive deficits & 1.40 & 0.63 & 4.91 & 1.00 & $0.03^{*}$ & 4.06 & $1.18-14.04$ \\
Depressive symptoms & -18.82 & $7,720.98$ & 0.00 & 1.00 & 1.00 & 0.00 & 0.00 \\
Frailty symptoms & 1.35 & 0.78 & 2.96 & 1.00 & 0.09 & 3.84 & $0.83-17.76$ \\
Age & 0.01 & 0.05 & 0.09 & 1.00 & 0.77 & 1.01 & $0.92-1.12$ \\
General anesthesia & 1.19 & 0.72 & 2.73 & 1.00 & 0.10 & 3.30 & $0.80-13.57$ \\
Constant & -5.43 & 4.14 & 1.72 & 1.00 & 0.19 & 0.00 & \\
\hline
\end{tabular}

Notes: \# Compared to local anesthesia under conscious sedation. ${ }^{*} p$ significance, $p<0.05$. Without SMARTIE: The model $\left(\chi^{2}(5)=13.3, p=0.02\right)$, explained I5.9\% $\left(\right.$ Nagelkerke $\left.R^{2}\right)$ of the variance in predicting mortality, correctly classifying $94.4 \%$ of cases. It was a good fit to the data $(p=0.31)$ on the Hosmer \& Lemeshow test of the goodness of fit.

including the use of MGA-based scores and the use of frailty scales as predictors of mortality and post-procedural complications. ${ }^{9,31}$ Comprehensive geriatric assessments in patients undergoing elective surgery have been shown to improve survival rates in hospitalized older adults as well as the identification of patients at greater risk for mortality and adverse in-hospital events. ${ }^{9,31-33}$ Geriatric assessment prior to TAVI may be particularly important as preexisting co-morbidities are predictive of poor outcomes in this population and may help improve the identification of older patients who benefit most from a TAVI. ${ }^{34}$ This study explored a MGA of TAVI patients with a holistic approach emphasizing both physical and neuropsychiatric risk factors. These data are potentially useful to inform decision-making for patient selection and for periprocedural management in collaboration with a team of psychiatrists and geriatricians.

In this study, the use of a screening tool increased identification of those at risk for cognitive deficits, depressive symptoms, and frailty (Table 1). These findings suggest that a structured screening tool such as SMARTIE could assist in the identification of geriatric symptoms as these may be easily missed in older patients being considered for TAVI unless they are actively screened for. This is important as cognitive deficits are critical predictors of functional decline, lack of mobility, and poor quality of life in older adults. ${ }^{35,36}$ While risks associated with more advanced stages of dementia or Alzheimer's disease are more commonly identified, subtle cognitive deficits or mild cognitive impairment are under-recognized and may still increase the risk of poorer post-procedural outcomes. ${ }^{37}$

Data from the SMARTIE cohort show that while almost all patients identified with depressive and frailty symptoms were subsequently diagnosed with depression and physical frailty, only $60 \%$ of those with cognitive deficits had clinical diagnoses of mild cognitive impairment or dementia following SMARTIE. This difference likely reflects practical limitations as not all of the patients screening positive for cognitive deficits could be seen in the memory disorders clinic prior to TAVI. There were several reasons for this, including the inability to schedule timely assessments before a planned TAVI as well as patients refusing to undergo cognitive assessments given their denial of deficits and lack of appreciation of the value of cognitive assessments. Also, the sheer burden of assessments prior to TAVI including the clinical review of cardiac conditions, laboratory visits, and pre-operative assessments likely dissuaded patients from seeing a geriatric psychiatrist. 
Study findings also demonstrated that symptoms of cognitive deficits predicted POD and mortality following TAVI, suggesting that it may be worthwhile to identify patients with subtle cognitive deficits prior to TAVI in addition to those with clinical diagnoses of mild cognitive impairment/ dementia. These findings resonate with the arguments of a recent study that stated that although dementia when treated, is not considered a contraindication to TAVI, undiagnosed mild cognitive impairment may be associated with potentially life-threatening complications. Findings in this study emphasize the need to identify and treat patients prior to TAVI to improve post-TAVI outcomes. ${ }^{38}$ Depressive symptoms and frailty were not independent predictors of POD or 30-day mortality, although these risk factors as previously documented have been associated with poor TAVI outcomes including delirium and cerebrovascular events. ${ }^{21,39}$

The regression models used to predict POD and 30day mortality used both SMARTIE and pre-SMARTIE patient cohorts pooled together in the analyses with the assumption that while the degree of true underlying deficits was similar between the groups, SMARTIE improved detection of them. Age and the use of general anesthesia were controlled for in the regression models to account for any differences between the groups, although other potentially limiting factors such as a number of baseline comorbidities, surgical risk scores, and TAVI urgency could not be analyzed due to lack of reliably available data from the pre-SMARTIE cohort. In addition, another limitation was that BMI was not collected and thus could not be adjusted for in the regression, although we did assess frailty, which has been closely related with both BMI and adverse outcomes ${ }^{40,41}$ Total delirium and mortality rates in this study were low but comparable to incidences of delirium (12-17\%) and 30-day mortality rates $(5-7 \%)$ previously reported in the literature for transfemoral TAVI. ${ }^{11,42,43}$ This may have been due to changes in clinical practice in the increased use of local vs general anesthesia in the SMARTIE group which may have led to improved TAVI outcomes including lower delirium rate and shorter hospital length of stay. In line with this, the pre-SMARTIE cohort underwent general anesthesia almost exclusively whereas the SMARTIE cohort employed local anesthesia with conscious sedation at a significantly higher rate. Therefore, we have controlled for the use of general anesthesia in our multivariable analysis as this change in clinical practice likely contributed to post-TAVI outcomes.
Another important potential methodological limitation of the study was the choice of instruments used to screen for frailty. Frailty symptoms were screened using three items encompassing the frailty screen: the ADL inventory, the TUG test for gait speed and hand grip strength test. Disability, defined as inability to perform specific ADLs without help, has been linked with low physical activity and slowness and was hence a suitable measure for frailty in this population. ${ }^{44}$ Poor hand grip strength and gait speed (measured using the TUG test) have also been demonstrated as predictors of future ADL disability in community-dwelling older people. ${ }^{26,27}$ However, due to practical limitations during TAVI consultations, not all patients could complete all three components of the frailty screen which may have led to under estimation of frailty in this study. Patients encountered several barriers to completing all frailty assessments including low mobility, risk of falls, and time constraints. While the ADL inventory, TUG test, and hand grip strength test are validated and reliable frailty assessments, the use of alternative frailty measures may address the aforementioned barriers. ${ }^{26,27}$ For example, prospectively screening for the upper-extremity function has been found to be a low-cost, time-efficient method for predicting hospital re-admission rates. ${ }^{45}$ Furthermore, a novel self-report questionnaire for measuring frailty has shown high agreement with performance-based walking and grip strength tests. ${ }^{46}$ Future research may utilize more feasible frailty measures that can be easily administered to bed-bound patients to address these limitations. Lastly, evidence for cognitive deficits, depressive symptoms, and frailty as well as post-operative outcomes were systematically extracted from the chart review by three authors for the pre-SMARTIE group. While we acknowledge that this is a potential confounder that may have introduced unintended bias in the data collection process, we attempted to reduce this risk by utilizing a template to extract data in a standardized procedure.

\section{Conclusion}

This study emphasizes the need to identify geriatric risk factors that impact post-procedural patient management. While it may not always be feasible to screen for patients' subtle deficits by incorporating extensive cognitive and neuropsychiatric batteries into routine cardiac assessments, a simple geriatric screen like the Mini-Cog used in this study may inform patient selection by identifying individuals at risk for cognitive deficits and subsequent poor outcomes. Moreover, screening for geriatric risk factors 
has the potential to contribute to target clinical care and improve health outcomes by the establishment of a multidisciplinary referral system for more comprehensive geriatric assessments and better management and treatment of risk factors in TAVI.

\section{Ethics}

This study was conducted in accordance with the Declaration of Helsinki and implemented with approval and oversight from the Research Ethics Board of Sunnybrook Health Sciences Centre. All participants provided written, informed consent.

\section{Acknowledgments}

This study has been funded by the Sunnybrook AFP Innovation fund [SHS-15-008]. N. Herrmann and KL Lanctôt were the principal investigators on the study.

\section{Author contributions}

All authors contributed to data analysis, drafting and revising the article, gave final approval of the version to be published, and agree to be accountable for all aspects of the work.

\section{Disclosure}

SE Fremes is supported by grant funding from Medtronic Inc. (Physio-Control Intl. Corp.), Bayer Pharmaceuticals, Bayer Inc. Germany, and HLT Inc., USA. HC Wijeysundera is supported by grant funding from Medtronic Inc and Edwards Life Sciences Inc, outside the submitted work. KL Lanctôt reports grants from the National Institute of Aging (5R01AG052510-02), Alzheimer's Association (PTC-18-543823), Canadian Institutes of Health Research (FRN\#308227), personal fees from AbbVie, Axovant Sciences Ltd, and Otsuka, outside the submitted work. The authors report no other conflicts of interest in this work.

\section{References}

1. Kappetein AP, Head SJ, Genereux P, et al. Updated standardized endpoint definitions for transcatheter aortic valve implantation: the Valve Academic Research Consortium-2 consensus document. J Thorac Cardiovasc Surg. 2013;145:6-23. doi:10.1016/j.jtcvs.2012.09.002

2. Ponikowski P, Voors AA, Anker SD, et al. ESC guidelines for the diagnosis and treatment of acute and chronic heart failure. Eur J Heart Fail. 2016;2016(18):891-975.

3. Elmalem S, Dumonteil N, Marcheix B, et al. Health-related quality of life after transcatheter aortic valve implantation in elderly patients with severe aortic stenosis. J Am Med Dir Assoc. 2014;15:201-206. doi:10.1016/j.jamda.2013.11.010
4. Ak A, Porokhovnikov I, Kuethe F, Schulze PC, Noutsias M, Schlattmann P. Transcatheter vs. surgical aortic valve replacement and medical treatment: systematic review and meta-analysis of randomized and non-randomized trials. Herz. Epub 2017 April 27.

5. Kilic T, Yilmaz I. Transcatheter aortic valve implantation: a revolution in the therapy of elderly and high-risk patients with severe aortic stenosis. J Geriatr Cardiol. 2017;14:204-217. doi:10.11909/j.issn.1671-5411.2017.03.002

6. Sardar P, Kundu A, Chatterjee S, et al. Transcatheter versus surgical aortic valve replacement in intermediate-risk patients: evidence from a meta-analysis. Catheter Cardiovasc Interv. 2017. doi:10.1002/ccd.27041

7. Nashef SA, Roques F, Sharples LD, et al. EuroSCORE II. Eur $J$ Cardiothorac Surg. 2012;41:734-744; discussion 744-735. doi:10.1093/ejcts/ezs043

8. Shahian DM, O'Brien SM, Filardo G, et al. Society of thoracic surgeons quality measurement task $\mathrm{F}$. The society of thoracic surgeons 2008 cardiac surgery risk models: part 3-valve plus coronary artery bypass grafting surgery. Ann Thorac Surg. 2009;88:S43-S62. doi:10.1016/j.athoracsur.2009.05.055

9. Stortecky S, Schoenenberger AW, Moser A, et al. Evaluation of multidimensional geriatric assessment as a predictor of mortality and cardiovascular events after transcatheter aortic valve implantation. JACC Cardiovasc Interv. 2012;5:489-496. doi:10.1016/j.jcin.2012.02.012

10. Sanchez E, Vidan MT, Serra JA, Fernandez-Aviles F, Bueno H. Prevalence of geriatric syndromes and impact on clinical and functional outcomes in older patients with acute cardiac diseases. Heart. 2011;97:1602-1606. doi:10.1136/hrt.2011.227504

11. Tse L, Bowering JB, Schwarz SK, Moore RL, Burns KD, Barr AM. Postoperative delirium following transcatheter aortic valve implantation: a historical cohort study. Can J Anaesth. 2015;62:22-30. doi:10.1007/s12630-014-0254-2

12. Mehta KY, Yaffe K, Langa KM, Sands L, Whooley MA, Covinsky KE. Additive effects of cognitive function and depressive symptoms on mortality in elderly community-living adults. J Gerontol A Biol Sci Med Sci. 2003;58:461-467.

13. Oldham MA, Vachon J, Yuh D, Lee HB. Cognitive outcomes after heart valve surgery: a systematic review and meta-analysis. $J \mathrm{Am}$ Geriatr Soc. 2018. doi:10.1111/jgs.15601

14. Dillon C, Machnicki G, Serrano CM, Rojas G, Vazquez G, Allegri RF. Clinical manifestations of geriatric depression in a memory clinic: toward a proposed subtyping of geriatric depression. $J$ Affect Disord. 2011;134:177-187. doi:10.1016/j.jad.2011.05.036

15. Alosco ML, Spitznagel MB, Cohen R, et al. Reduced cerebral perfusion predicts greater depressive symptoms and cognitive dysfunction at a 1-year follow-up in patients with heart failure. Int $J$ Geriatr Psychiatry. 2014;29:428-436. doi:10.1002/gps.4023

16. Burkauskas J, Brozaitiene J, Bunevicius A, Neverauskas J, Zaliunaite V, Bunevicius R. Association of depression, anxiety, and type D personality with cognitive function in patients with coronary artery disease. Cogn Behav Neurol. 2016;29:91-99. doi:10.1097/ WNN.0000000000000093

17. Drudi LA, Turkdogan MS, Huynh C, et al. Association of depression with mortality in older adults undergoing transcatheter or surgical aortic valve replacement. JAMA. 2018;3:191-197.

18. Kano S, Yamamoto M, Shimura T, et al. Gait speed can predict advanced clinical outcomes in patients who undergo transcatheter aortic valve replacement: insights from a Japanese multicenter registry. Circ Cardiovasc Interv. 2017;10. doi:10.1161/ CIRCINTERVENTIONS.117.005088

19. Okoh AK, Chauhan D, Kang N, et al. The impact of frailty status on clinical and functional outcomes after transcatheter aortic valve replacement in nonagenarians with severe aortic stenosis. Catheter Cardiovasc Interv. 2017. doi:10.1002/ccd.27083

20. Shimura T, Yamamoto M, Kano S, et al. Impact of the clinical frailty scale on outcomes after transcatheter aortic valve replacement. Circulation. 2017;135:2013-2024. doi:10.1161/CIRCULATIONAHA.116.025630 
21. Anand A, Harley C, Visvanathan A, et al. The relationship between preoperative frailty and outcomes following transcatheter aortic valve implantation: a systematic review and meta-analysis. Eur Heart J Qual Care Clin Outcomes. 2017;3:123-132. doi:10.1093/ehjqcco/qcw030

22. Borson S, Scanlan JM, Watanabe J, Tu SP, Lessig M. Improving identification of cognitive impairment in primary care. Int $J$ Geriatr Psychiatry. 2006;21:349-355. doi:10.1002/gps.1470

23. Borson S, Scanlan J, Brush M, Vitaliano P, Dokmak A. The mini-cog: a cognitive 'vital signs' measure for dementia screening in multilingual elderly. Int J Geriatr Psychiatry. 2000;15:1021-1027.

24. Li C, Friedman B, Conwell Y, Fiscella K. Validity of the Patient Health Questionnaire 2 (PHQ-2) in identifying major depression in older people. J Am Geriatr Soc. 2007;55:596-602. doi:10.1111/ j.1532-5415.2007.01103.x

25. Kroenke K, Spitzer RL, Williams JB. The Patient Health Questionnaire-2: validity of a two-item depression screener. Med Care. 2003;41:1284-1292. doi:10.1097/01.MLR.0000093487.78664.3C

26. Apostolo J, Cooke R, Bobrowicz-Campos E, et al. Predicting risk and outcomes for frail older adults: an umbrella review of frailty screening tools. JBI Database System Rev Implement Rep. 2017;15:1154-1208. doi:10.11124/JBISRIR-2016-003018

27. Vermeulen J, Neyens JC, van Rossum E, Spreeuwenberg MD, de Witte LP. Predicting ADL disability in community-dwelling elderly people using physical frailty indicators: a systematic review. $B M C$ Geriatr. 2011;11:33. doi:10.1186/1471-2318-11-33

28. Nishimura K, Yokoyama K, Yamauchi N, et al.; Investigators $\mathrm{T}$. Sensitivity and specificity of the Confusion Assessment Method for the Intensive Care Unit (CAM-ICU) and the Intensive Care Delirium Screening Checklist (ICDSC) for detecting post-cardiac surgery delirium: a single-center study in Japan. Heart Lung. 2016;45:15-20. doi:10.1016/j.hrtlng.2015.11.001

29. Maas EH, Pieters BM, Van de Velde M, Rex S. General or Local Anesthesia for TAVI? A systematic review of the literature and meta-Analysis. Curr Pharm Des. 2016;22:1868-1878.

30. Soundhar A, Udesh R, Mehta A, et al. Delirium following transcatheter aortic valve replacement: national inpatient sample analysis. J Cardiothorac Vasc Anesth. 2017. doi:10.1053/j.jvca.2017.03.016

31. Kamga M, Boland B, Cornette P, et al. Impact of frailty scores on outcome of octogenarian patients undergoing transcatheter aortic valve implantation. Acta Cardiol. 2013;68:599-606. doi:10.2143/ AC.68.6.8000007

32. Ellis G, Whitehead MA, Robinson D, O`Neill D, Langhorne P. Comprehensive geriatric assessment for older adults admitted to hospital: meta-analysis of randomised controlled trials. BMJ. 2011;343:d6553. doi:10.1136/bmj.d6553

33. Kim KI, Park KH, Koo KH, Han HS, Kim CH. Comprehensive geriatric assessment can predict postoperative morbidity and mortality in elderly patients undergoing elective surgery. Arch Gerontol Geriatr. 2013;56:507-512. doi:10.1016/j.archger.2012.09.002
34. Guggiari C, Locca D, Smith C, Monney P, Vogt P, Bula C. [Geriatric assessment in old patients candidate to transcatheter aortic implantation]. Rev Med Suisse. 2013;9:2040-2043.

35. Jefferson AL, Cahn-Weiner D, Boyle P, et al. Cognitive predictors of functional decline in vascular dementia. Int $J$ Geriatr Psychiatry. 2006;21:752-754. doi:10.1002/gps.1556

36. Newman MF, Grocott HP, Mathew JP, et al., Neurologic Outcome Research G, the Cardiothoracic Anesthesia Research Endeavors Investigators of the Duke Heart C. Report of the substudy assessing the impact of neurocognitive function on quality of life 5 years after cardiac surgery. Stroke. 2001;32:2874-2881.

37. Alagiakrishnan K, Marrie T, Rolfson D, et al. Simple cognitive testing (Mini-Cog) predicts in-hospital delirium in the elderly. $\mathrm{J}$ Am Geriatr Soc. 2007;55:314-316. doi:10.1111/j.1532-5415.2007.01058.x

38. Damier E, Chidlovskii E, Bertrand B, Dang VM, Vanzetto G, Couturier P. [Multidimensional geriatric assessment before transcatheter aortic valve implantation in frail elderly patients with one-year follow-up. Cardio-geriatrician collaboration benefits?]. Ann Cardiol Angeiol (Paris). 2016;65:250-254. doi:10.1016/j.ancard.2016.05.001

39. Assmann P, Kievit P, van der Wulp K, et al. Frailty is associated with delirium and mortality after transcatheter aortic valve implantation. Open Heart. 2016;3:e000478. doi:10.1136/openhrt-2016-000478

40. Hubbard RE, Lang IA, Llewellyn DJ, Rockwood K. Frailty, body mass index, and abdominal obesity in older people. J Gerontol A Biol Sci Med Sci. 2010;65:377-381. doi:10.1093/gerona/glp186

41. Makary MA, Segev DL, Pronovost PJ, et al. Frailty as a predictor of surgical outcomes in older patients. $J$ Am Coll Surg. 2010;210:901-908. doi:10.1016/j.jamcollsurg.2010.01.028

42. Giordana F, D‘Ascenzo F, Nijhoff F, et al. Meta-analysis of predictors of all-cause mortality after transcatheter aortic valve implantation. Am J Cardiol. 2014;114:1447-1455. doi:10.1016/j. amjcard.2014.07.081

43. Tamburino $\mathrm{C}$, Capodanno $\mathrm{D}$, Ramondo $\mathrm{A}$, et al. Incidence and predictors of early and late mortality after transcatheter aortic valve implantation in 663 patients with severe aortic stenosis. Circulation. 2011;123:299-308. doi:10.1161/CIRCULATIONAHA.110.946533

44. Provencher V, Beland F, Demers L, et al. Are frailty components associated with disability in specific activities of daily living in community-dwelling older adults? A multicenter Canadian study. Arch Gerontol Geriatr. 2017;73:187-194. doi:10.1016/j. archger.2017.07.027

45. Ehsani H, Mohler MJ, Golden T, Toosizadeh N. Upper-extremity function prospectively predicts adverse discharge and all-cause COPD readmissions: a pilot study. Int J Chron Obstruct Pulmon Dis. 2019;14:39-49. doi:10.2147/COPD.S182802

46. Op Het Veld LPM, de Vet HCW, van Rossum E, Kempen G, van Kuijk SMJ, Beurskens A. Substitution of Fried's performance-based physical frailty criteria with self-report questions. Arch Gerontol Geriatr. 2018;75:91-95. doi:10.1016/j.archger.2017.11.009
Clinical Interventions in Aging

\section{Publish your work in this journal}

Clinical Interventions in Aging is an international, peer-reviewed journal focusing on evidence-based reports on the value or lack thereof of treatments intended to prevent or delay the onset of maladaptive correlates of aging in human beings. This journal is indexed on PubMed Central, MedLine, CAS, Scopus and the Elsevier
Bibliographic databases. The manuscript management system is completely online and includes a very quick and fair peer-review system, which is all easy to use. Visit http://www.dovepress.com/ testimonials.php to read real quotes from published authors. 